\title{
MELHORA DA QUALIDADE DE VIDA APÓS O TRANSPLANTE RENAL EM COMPARAÇÃO COM O PERÍODO DIALÍTICO: UM ESTUDO EXPLORATÓRIO
}

\author{
Improvement of quality of life after renal transplantation in comparison to dialysis: an exploratory \\ study
}

\author{
Camila Lima, ${ }^{1}$ Maria Aparecida Fadil Romão, ${ }^{2}$ Igor Denizarde Bacelar Marques,${ }^{3}$ Carmen Mohamad Rida Saleh,${ }^{2}$ \\ Elisa Midori Yagyu, ${ }^{3}$ Mariana de Freitas Grassi. ${ }^{1}$
}

\begin{abstract}
RESUMO
Objetivo: Avaliar a qualidade de vida dos pacientes portadores de doença renal crônica após o transplante renal. Método: Trata-se de um estudo transversal, exploratório, que foi realizado em 50 pacientes, utilizando o instrumento que avalia a qualidade de vida, o Medical Outcomes Study 36 Item Short Form Health Survey (SF-36) e variável de classificação econômica após o transplante renal. O resultado do estudo foi comparado com 50 pacientes em hemodiálise, utilizando o mesmo instrumento. Resultados: A mediana dos resultados do SF-36 foram: capacidade funcional 92, aspectos físicos 100 , dor 74 , estado geral de saúde 63 , vitalidade 70 , aspectos sociais 87 , aspectos emocionais 100 e saúde mental 76. Comparando estes resultados com os obtidos em pacientes em hemodiálise, houve melhora na capacidade funcional (92 vs. 80; $\mathrm{p}<0,05)$. Os demais componentes do SF-36 foram semelhantes entre pacientes transplantados e dialíticos. Conclusão: Pacientes transplantados renais apresentam melhor qualidade de vida, expressa pelo domínio capacidade funcional do escore SF-36, do que pacientes com doença renal crônica em tratamento dialítico.
\end{abstract}

Descritores: Qualidade de Vida; Transplante de Rim; Indicadores de Qualidade de Vida.

Instituições:

${ }^{1}$ Unidade de Diálise do Serviço de Nefrologia do Hospital das Clínicas da Faculdade de Medicina da Universidade de São Paulo, São Paulo/SP

${ }^{2}$ Divisão de Enfermagem do Hospital das Clínicas da Faculdade de Medicina da Universidade de São Paulo, São Paulo/SP

${ }^{3}$ Serviço de Transplante Renal do Hospital das Clínicas da Universidade de São Paulo, São Paulo/SP

\section{Correspondência:}

Camila Lima

Unidade de Diálise Agudos - Av. Dr. Enéas de Carvalho Aguiar, 255 -

$7^{\mathrm{a}}$ andar - CEP 05403900, São Paulo/SP

Tel: (11) 2661-6291

E-mail: camilaxlima@gmail.com

Recebido em: 28.07.2011

Aceito em: 29.08.11

\section{INTRODUÇÃO}

O transplante renal é a terapia de escolha para pacientes com doença renal crônica (DRC). Trata-se de um procedimento que pode ser realizado com doadores vivos aparentados (até o quarto grau de consanguinidade ou cônjuges) e falecidos. Doadores vivos não aparentados necessitam de avaliação psico-social, aprovação de um comitê de ética independente e autorização judicial para serem aceitos.

Houve um aumento no número de transplantes renais no Brasil, tanto de doadores vivos 6,7 por milhão de pessoas (pmp), em 1998, para 8,6 pmp em 2010, quanto em doadores falecidos $3,8 \mathrm{pmp} \mathrm{em}$ 1999 versus 9,9 pmp em 2010. ${ }^{1}$

Considerando o custo do transplante renal no contexto prétransplante renal, pode ter havido economia de $34 \%$ em dois anos. ${ }^{2}$

Apesar dessas assertivas, o tempo de espera para o transplante renal com doador falecido em pacientes adultos nos Estados Unidos da América (EUA) em 2004 foi em média 2,85 anos, tendo aumentado em 2006 para 4,58 anos. ${ }^{3}$ Em 2010 no Brasil, cerca de 34,640 pessoas aguardavam um transplante renal. ${ }^{1}$

A qualidade de vida é um conceito muito utilizado, porém só a partir da década de 1970 vem sendo estudada de maneira substancial, por ser considerada um termo subjetivo e multidimensional havendo dificuldades em sua aplicação e mensuração. ${ }^{4}$ 
Definição de qualidade de vida pela Organização Mundial de Saúde (OMS):

"A percepção do indivíduo de sua posição na vida no contexto da cultura e sistema de valores nos quais ele vive e em relação aos seus objetivos, expectativas, padrões e preocupações." ${ }^{5}$

O Medical Outcomes Study 36 Item Short Form Health Survey (SF-36) é um questionário genérico de qualidade de vida validado internacionalmente, com oito conceitos de saúde, construído para satisfazer o mínimo dos padrões psicométricos necessários para fazer comparações entre os grupos. A experiência com esse instrumento tem sido documentada em cerca de 4.000 publicações. ${ }^{6}$ É compreensível que o transplante renal bem sucedido renove a vida do paciente portador de DRC. Apesar de ser amplamente aceita, essa assertiva tem sido pouco analisada na literatura. ${ }^{7}$ É visível observar que o transplante renal não é via final de tratamento nem cura definitiva de patologia, portanto é um tratamento que visa a busca de melhor qualidade de vida, mas isso não o abstêm da terapia medicamentosa (imunossupressores), supervisão médica, risco de rejeição, o potencial de re-hospitalização, risco de infecção e as alterações na aparência física que lhe trazem muitas consequências. ${ }^{8,9}$

O objetivo do estudo foi avaliar a qualidade de vida de pacientes portadores de DRC após transplante renal, utilizando dados socioeconômicos e o SF36.

\section{MÉTODOS}

\section{Desenho do Estudo}

Trata-se de um estudo transversal, exploratório. A pesquisa foi aplicada utilizando-se os critérios da Associação Brasileira de Empresas e Pesquisa (ABEP) para a classificação do nível econômico e o questionário de qualidade de vida SF- 36 em pacientes no póstransplante renal. O critério de classificação econômica (ABEP) estima o poder de compra das pessoas, abandonando a pretensão de classificar a população em termos de "classes sociais".

As classes econômicas são avaliadas em formulário próprio, com um sistema de pontos (0-34) que consiste em: posse de itens (0-4) e grau de instrução do chefe da família (0-5). O resultado é classificado gradativamente da maior para a menor classe econômica: A1, A2, B1, B2, C, D e E. ${ }^{10}$

O SF 36 inclui um item de multi-escala que avalia oito conceitos de saúde:

1. restrição das atividades físicas devido a problemas de saúde;

2. limitações em atividades sociais devido a problemas físicos ou emocionais;

3. limitações usuais no papel devido a atividade física e problemas de saúde;

4. dor corporal;

5. aspectos gerais de saúde mental (bem estar e sofrimento psíquico);

6. limitações em papel atividades habituais devido a problemas emocionais;

7. vitalidade (energia e fadiga)

8. percepção geral de saúde. ${ }^{11}$

O cálculo do SF-36 foi obtido nos oito componentes utilizando o
Raw Scale em notas de oito domínios, que variam de 0 (zero) a 100 (cem), onde $0=$ pior e $100=$ melhor para cada domínio. A mediana dos resultados em cada componente do SF-36 dos pacientes transplantados foi comparada com a população em diálise no mesmo hospital.

\section{Local do Estudo}

Este estudo foi realizado no Serviço de Transplante Renal do Hospital das Clínicas da Faculdade de Medicina da Universidade de São Paulo, uma vez que esses pacientes realizavam acompanhamento médico de rotina.

Em sua maioria, os pacientes não apresentavam problemas de ordem física, resultando em melhor análise dos dados do estudo.

\section{Amostra}

Foram admitidos para este estudo, 50 pacientes, no período de julho a outubro de 2009 .

\section{Critérios para inclusão:}

- Pacientes que se submeteram a transplante renal há, no mínimo, um ano;

- Pacientes com idade igual ou maior que 18 anos;

- Pacientes que concordaram e possuíam nível cognitivo adequado para compreender e responder o instrumento utilizado.

\section{Aspectos Éticos}

Este estudo foi procedido da aprovação pela Comissão de Pareceristas dos Projetos de Pesquisa do Curso de Especialização Instituto Central do Hospital das Clinicas da Faculdade de Medicina da Universidade de São Paulo (ICHCFMUSP). Antes do início da apresentação do instrumento de pesquisa, foi explicado aos pacientes selecionados o objetivo deste trabalho, sendo enfatizado que sua recusa não implicaria em qualquer prejuízo ao seu tratamento.

\section{Operacionalização do Estudo}

Foram selecionados os pacientes que estavam dentro dos critérios exigidos e que estavam com consulta agendada no Ambulatório do Serviço de Transplante Renal do Hospital das Clinicas da Faculdade de Medicina da Universidade de São Paulo, no período da coleta.

O local para abordagem e coleta foi a sala de enfermagem, onde os pacientes transplantados, entre outros, realizavam aferição de pressão arterial e medidas antropométricas antes da consulta médica.

Após explicação sobre o objetivo do estudo e da necessidade de preenchimento de um questionário $\mathrm{ABEP}$ e de qualidade de vida SF-36, os pacientes que concordaram assinaram o termo de consentimento livre e esclarecido.

Durante o preenchimento do questionário, quaisquer eventuais dúvidas sobre ele eram esclarecidas aos pacientes. Não foi estabelecido qualquer tempo limite para devolução do instrumento.

\section{Variáveis do Estudo}

Foram selecionadas variáveis referentes a características sóciodemográficas: sexo, idade, escolaridade, estado civil (casado, solteiro, divorciado e viúvo) e grau de instrução. 


\section{Análise Estatística}

As variáveis do SF36 foram expressas em forma de medianas, intervalo interquartil e variações, dada a predominância de distribuição não-paramétrica entre os resultados. Para comparação entre os resultados do grupo de pacientes transplantados com o grupo de pacientes em diálise, foi utilizado o teste de MannWhitney. Significância estatística foi assumida para valores de $\mathrm{p}<$ 0,05. A análise estatística foi realizada com o programa Statistical Package for the Social Sciences, versão 17.0 (SPSS Inc., Chicago, IL, USA).

\section{RESULTADOS}

Da população estudada, $56 \%$ havia sido transplantada com doadores vivos. O tempo de seguimento médio foi de sete anos variando de um a 14 anos para transplantados com doador vivo e de um a 20 anos para os transplantados com doador falecido.

Foram encontrados 21 (42\%) pacientes do sexo feminino e 29 (58\%) do sexo masculino; com um predomínio de $8 \%$ na população masculina. A média de idade dos pacientes foi de 41 anos, sendo a idade mínima de 20 anos e a máxima de 69 anos.

Os níveis de escolaridade dos pacientes estudados foram; ensino fundamental incompleto 22 (44\%), representando praticamente a metade, ensino fundamental $5(10 \%)$; apenas $12(24 \%)$ cursaram o ensino médio e $10(20 \%)$ com ensino superior ou cursando e um paciente $(03 \%)$ com pós graduação.

Em relação ao estado civil, a maioria dos pacientes era representada por solteiros (22 - 44\%) e casados (22 -44\%); sendo que os restantes eram desquitados $(03-06 \%)$ e viúvos $(03-06 \%)$.

Na classificação de acordo com o nível econômico, ABEP, as classes predominantes foram $\mathrm{B}$ e $\mathrm{C}$, seguidas de $\mathrm{De} \mathrm{E}$, e, minoritariamente, A. Os valores percentuais foram os seguintes: A1 (2\%), A2 (2\%), $\mathrm{B} 1(22 \%), \mathrm{B} 2(20 \%), \mathrm{C} 1(22 \%), \mathrm{C} 2(6 \%), \mathrm{D}(18 \%)$ e E (8\%).

Tabela 1: Mediana dos valores obtidos para cada componente do SF36 nos 50 pacientes estudados.

\begin{tabular}{lccc}
\hline $\begin{array}{l}\text { Componentes } \\
\text { do SF-36 }\end{array}$ & Mediana & $\begin{array}{c}\text { Intervalo } \\
\text { interquartil }\end{array}$ & Variação \\
\hline $\begin{array}{l}\text { Capacidade } \\
\text { Funcional }\end{array}$ & 92 & 26 & $15-100$ \\
$\begin{array}{l}\text { Aspectos } \\
\text { físicos }\end{array}$ & 100 & 75 & $0-100$ \\
$\begin{array}{l}\text { Dor } \\
\begin{array}{l}\text { Estado geral } \\
\text { da saúde }\end{array}\end{array}$ & 74 & 38 & $31-100$ \\
$\begin{array}{l}\text { Vitalidade } \\
\text { Aspectos }\end{array}$ & 70 & 33 & $15-95$ \\
sociais & 87 & 35 & $15-100$ \\
$\begin{array}{l}\text { Aspectos } \\
\text { emocionais }\end{array}$ & 100 & 50 & $12-100$ \\
Saúde mental & 76 & 67 & $0-100$ \\
\hline
\end{tabular}

O presente estudo foi comparado ao estudo realizado na Unidade de Diálise do Serviço de Nefrologia do Hospital das Clínicas da Faculdade de Medicina da Universidade de São Paulo utilizandose o questionário de qualidade de vida SF-36 em 50 pacientes submetidos à terapia de substituição renal (hemodiálise). ${ }^{12}$
Tabela 2. Comparação entre 50 pacientes transplantados e 50 pacientes em hemodiálise do Hospital das Clinicas da Faculdade de Medicina da Universidade de São Paulo (2006). ${ }^{12}$

\begin{tabular}{|l|c|c|c|}
\hline $\begin{array}{l}\text { Componentes } \\
\text { do SF-36 }\end{array}$ & $\begin{array}{c}\text { Pacientes } \\
\text { Transplantados }\end{array}$ & $\begin{array}{c}\text { Pacientes em } \\
\text { Diálise }\end{array}$ & $\boldsymbol{p}$ \\
\hline $\begin{array}{l}\text { Capacidade } \\
\text { funcional }\end{array}$ & 92 & 80 & 0,0009 \\
\hline $\begin{array}{l}\text { Aspectos } \\
\text { físicos }\end{array}$ & 100 & 87 & 0,66 \\
\hline Dor & 74 & 69 & 0,26 \\
\hline $\begin{array}{l}\text { Estado geral } \\
\text { da saúde }\end{array}$ & 63 & 56 & 0,12 \\
\hline Vitalidade & 70 & 64 & 0,14 \\
\hline $\begin{array}{l}\text { Aspectos } \\
\text { sociais }\end{array}$ & 87 & 88 & 0,35 \\
\hline $\begin{array}{l}\text { Aspectos } \\
\text { emocionais }\end{array}$ & 100 & 100 & 0,22 \\
\hline Saúde mental & 76 & 63 & 0,06 \\
\hline
\end{tabular}

\section{DISCUSSÃO}

A proporção obtida em relação ao gênero foi semelhante aos dados encontrados no Censo de Diálise da SBN (Sociedade Brasileira de Nefrologia), apresentando $57 \%$ dos pacientes em terapia renal substitutiva do gênero masculino, uma proporção próxima dos dados obtidos neste estudo. ${ }^{13}$

O resultado encontrado sobre a idade é semelhante ao do estudo de Pereira et al. que avaliaram a qualidade de vida após o transplante renal, sendo a idade média de $41 \pm 12$ anos, com idade mínima de 17 anos e a máxima de 66 anos.

Quando os dados de qualidade de vida obtidos no presente estudo foram comparados com pacientes em diálise avaliados no mesmo hospital, observamos escores mais elevados nos pacientes transplantados em seis dos oito componentes do SF-36: capacidade funcional, aspectos físicos, dor, estado geral de saúde, vitalidade e saúde mental. Os escores relativos aos aspectos emocionais mantiveram-se constantes entre os dois grupos. $\mathrm{O}$ aspecto social foi um pouco inferior nos pacientes transplantados (87) em relação aos pacientes em diálise (88). Essas diferenças, entretanto, não atingiram significância estatística.

Considerando-se os resultados não tão satisfatórios dos aspectos sociais, pode ser que apesar da restauração da função renal, muitos pacientes após o transplante continuam a se auto-identificar como deficientes. Quando receptores de rins ostentam o rótulo de deficiência, eles são menos propensos a participar em atividade de trabalho ou social. ${ }^{14}$

Apesar das diferenças acima descritas, apenas a capacidade funcional apresentou diferença estatisticamente significante entre os dois grupos comparados $(p=0,009)$, revelando uma melhora neste componente nos pacientes transplantados.

A qualidade de vida vem se tornando um parâmetro importante de resultado adicional para o transplante renal.

Uma recente metanálise realizada por Landreneau e colaboradores, onde foram analisados 16 estudos de medidas de qualidade de vida comparando hemodiálise e transplante renal, resultou que o 
transplante renal foi significativamente mais eficaz na melhoria de todos os três domínios (qualidade de vida geral, funcionamento físico e funcionamento psicossocial), particularmente qualidade de vida geral e funcionamento físico. ${ }^{15}$

Tonelli et al. selecionaram 110 estudos elegíveis com um total de 1.922.300 participantes. A maioria dos estudos encontrou mortalidade significantemente menor associada ao transplante, bem como magnitude relativa do beneficio pareceu aumentar ao longo do tempo $(\mathrm{p}<0,001){ }^{16}$

Esses resultados dos estudos validam as tentativas atuais de aumentar o número de pessoas no mundo inteiro que se beneficiam com o transplante renal.

\section{CONCLUSÃO}

Os pacientes transplantados renais apresentaram escores do SF-36 superiores aos dos urêmicos sob hemodiálise, demonstrando que o sucesso do transplante renal como terapia para a fase final da DRC alcançou seu objetivo de melhora e reabilitação física e mental, tanto para a qualidade e quantidade de vida que o transplante renal oferece.

\section{ABSTRACT}

Purpose: To assess the quality of life of persons suffering from chronic kidney disease after them having a kidney transplanted. Methods: It was a qualitative research free from explorative character which was conducted by using a tool to assess the quality of life named Medical Outcomes Study 36 item Short from Health Survey (SF36) and variable according to economical classes in 50 patients after kidney transplantation; results attained in the analysis when compared to patients under hemodialysis used the same instrument. Statistical analyzes were expressed as median, interquartile range and variations; for comparisons between groups, the Mann-Whiteney was used. Results: Results of SF-36 were expressed as median: functional capacity (FC) 92, physical aspect (PA) 100, pain (P) 74, general healthy aspect (GHA) 63, vitality (V) 70, social aspects (SA) 87, emotional aspects (EA) 100, and mental health (MH) 76. When comparing the presented research to patients under hemodialysis, best rates were found in 6 out of 8 SF-36 compounds: FC, PA, P, GHA, V and MH. SE has remained constant in both groups and SS was lower in transplanted patients. There was statistical significance in FC with $\mathrm{p}<0,009$. Conclusion: Kidney transplanted recipients had SF-36 scores higher than patients under hemodialysis.

Keywords: Quality of Life; Kidney Transplantation; Indicators of Quality of Life

\section{REFERÊNCIAS}

1. Pestana JOM, Galante NZ, Silva AT, Harada KM, Garcia VD, Abbud-Filho M, Campos HH, Sabbaga E. O contexto do transplante renal no Brasil e sua disparidade geográfica. J Bras Nefrol. 2011;33(4):472-84.

2. Mc Pake D, Burnapp L. Caring for patients after kidney transplantation. Nurs Stand. 2009;23(19):49-57.

3. Butt Z,Yount SE, Caicedo JC, Abecassis MM, Cella D. Quality of life assessment in renal transplant: Review and future directions. Clin. Transplant. 2008;22(3):292-303.

4. Almeida, AM. Revisão: A importância da saúde mental na qualidade de vida e sobrevida do portador de insuficiência renal crônica. J Bras Nefrol. 2003;25(4):208-13.

5. Organização Mundial da Saúde. Divisão de saúde mental grupo WHOQOL 1994. Versão em português dos instrumentos de avaliação de qualidade de vida (WHOQOL) 1998. Obtido de http: // www.hcpa.vtrg.sbr/psiq/whoqol. html em 04/04/2009

6. Ciconelli RM. Tradução para o Português e validação do questionário de genérico de Avaliação de Qualidade de Vida [tese]. São Paulo: Título de Doutor em Medicina. Universidade Federal de São Paulo; 1997.

7. Pereira LC, Chang J, Romão MAF, Abensur H, Araújo MRT, Noronha IL, et al. Qualidade de vida relacionada a saúde em pacientes transplantado renal. J Bras Nefrol. 2003;25(1):10-6.

8. Mendes CA, Shiratori K. As percepções dos pacientes de transplante renal. Nursing. 2002;5(44):15-22.
9. Liu H, Feurer ID, Dwyer K, Speroff T, Shaffer D, Wright Pinson C. The effects of gender and age on health-related quality of life following kidney transplantation. J. Clin. Nurs. 2008;17(1):82-9.

10. Associação Brasileira das Empresas de Pesquisa [homepage na internet]. Critério de classificação econômica [acesso em 30 maio de 2010] Disponível em http: www.abep.org/novo/ContentID=301

11. Ware JEJr, Sherbourne CD. The MOS 36-Item Short-Form Helath Survey (SF - 36): I Conceptual Framework and Item Selection. Med Care. 1992;30(6):473-83.

12. Fadil Romão MA, Romão Junior JE, Belasco AGS, Barbosa DA. Qualidade de vida de pacientes com insuficiência renal crônica terminal em hemodiálise de alta eficiência. Rev Gaúcha de enferm. 2006;27(4):593-9.

13. Sesso R, Lopes AA, Thome FS, Bevilacqua JL, Junior JER, Lugon J. Relatório do Censo Brasileiro de Diálise. J Bras Nefrol. 2008;30(4):233-8.

14. McGee J, Jackson NR, Slakey DP. Disability and kidney transplantation in the United States. Clin Transplant. 2012; 26(3):377-81

15. Landreneau K, Lee K, Landreneau MD. Quality of life in patients undergoing hemodialysis and renal transplantation a meta-analytic review. Nephrol Nurs J. 2010 Jan-Feb;37(1):37-44.

16. Tonelli M, Wiede N, Knoll G, Bello A, Browne S, Jadhav D, Klarenbach S, Gill J. Systematic review: kidney transplantion compared with dialisis in clinically relevant outcomes. Am J Transplant. 2011; 11(10):2093-109. 